\title{
Exploring Ambient Air Quality Notifications for Smart Rooms
}

\author{
Chi Tai Dang, Ilhan Aslan, Andreas Seiderer, Elisabeth André \\ \{dang,andre\}@informatik.uni-augsburg.de,\{seiderer,aslan\}@hcm-lab.de \\ University of Augsburg \\ Universitätsstraße 6a, 86159 Augsburg, Germany
}

\begin{abstract}
Many regular meeting participants know the atmosphere of a meeting in a closed room when the air becomes stifling and uncomfortable. With increased concentration of carbon dioxide $\left(\mathrm{CO}_{2}\right)$, the cognitive performance of participants can also suffer as a result. Therefore, it makes sense to retrofit such meeting rooms with smarthome technology to improve the air quality. However, existing meeting rooms often do not have built-in ventilation systems. Hence, smart technology can be used to measure air quality and communicate that by ambient notifications such as ambient lighting. Actively ventilating a room is then left to the participants of a meeting. This paper reports on a user study demonstrating that the kind of unobtrusive notification from an ambient air quality display has a significant influence on response times of perceiving the information while being concentrated on the course of the meeting.
\end{abstract}

\section{CCS CONCEPTS}

- Human-centered computing $\rightarrow$ Interactive systems and tools; Empirical studies in ubiquitous and mobile computing; • Applied computing $\rightarrow$ Consumer health.

\section{KEYWORDS}

smart home, air quality, co2, ambient light, ambient notification, meeting room, smartwatch, user study

\section{ACM Reference Format:}

Chi Tai Dang, Ilhan Aslan, Andreas Seiderer, Elisabeth André. 2020. Exploring Ambient Air Quality Notifications for Smart Rooms. In 6th EAI International Conference on Smart Objects and Technologies for Social Good (GoodTechs '20), September 14-16, 2020, Antwerp, Belgium. ACM, New York, NY, USA, 6 pages. https://doi.org/10.1145/3411170.3411229

\section{INTRODUCTION}

In recent years, the smarthome market has developed at a tremendous pace and as a result has generated a rich variety of products. The high availability of low-priced commodity smarthome devices based on IoT $^{1}$-technologies led to an increasingly widespread use of such technologies. In most cases, already existing buildings with non-smart ordinary rooms and environments were incrementally equipped with smart technologies. This approach is applied not only to privately used buildings but also to business locations and

\footnotetext{
${ }^{1}$ Internet of Things

GoodTechs '20, September 14-16, 2020, Antwerp, Belgium

(C) 2020 Association for Computing Machinery.

This is the author's version of the work. It is posted here for your personal use. Not for redistribution. The definitive Version of Record was published in 6th EAI International Conference on Smart Objects and Technologies for Social Good (GoodTechs '20), September 14-16, 2020, Antwerp, Belgium, https://doi.org/10.1145/3411170.3411229.
}

spaces, such as a meeting room, which is the focus of this work. Meeting rooms serve to meet with several people and hold briefings, discussions, seminars, or workshops. During such meetings, the windows and doors of the rooms are usually closed in order to provide a silent and comfortable environment. However, in such a closed environment, air quality drops quickly with increasing numbers of participants, which reduces the participants' ability to concentrate. For example, gases emitted by people as they breathe, such as carbon dioxide $\left(\mathrm{CO}_{2}\right)$, accumulate over time in a room without people being able to perceive it directly. Higher concentrations of $\mathrm{CO}_{2}$ can have negative effects on cognitive performance or even lead to physiological symptoms $[2,4,14]$. Hence, it is important, in particular during longer meetings, to regularly assess the air quality of the room and, if necessary, to supply sufficient fresh air by suitable means such as a ventilation system. Smarthome technologies are ideally suited for such purposes, as there are many low-cost sensors for measuring air quality as well as the presence of people and for controlling actors of a ventilation system.

However, most meeting rooms that are retrofitted with smarthome technology are not equipped with a built-in ventilation system. Ventilation of such rooms can then only be achieved by manually opening windows and doors. In this case, smart technology can only achieve part of the goal, that is to determine air quality and communicate the need for ventilation. Since the participants of a meeting usually come together to work together, it would be inappropriate to disrupt the meeting with annoying notifications or even interrupt the work processes. Therefore, obtrusive notification types, such as a loudspeaker announcement, are not suitable ways of notification. Much better suited are ambient notifications such as ambient lighting or subtle light signals, as these can be perceived by participants through peripheral awareness $[10,21]$ and do not directly interrupt current work processes. With several participants in a meeting, however, it may happen that none of the participants actively takes care of ventilation, even though several of them have noticed the notification. This may have different reasons, for example because the notification was perceived late or because it was left to other participants. Here, the question arises whether the type of unobtrusive ambient notifications could have an influence on the motivation to actively take care of ventilation.

In order to explore questions that address this issue, we conducted a study in a real smart environment, that is a meeting room in a university building. The meeting room was previously equipped with smart technology to improve indoor air during meetings by continuously measuring air quality and displaying it through ambient lighting [19]. This allows the participants of a meeting to perceive the objectively measured air quality and, if necessary, improve the air quality by opening a window or taking a break when appropriate. In this paper, we present the results of a study that addresses whether different kinds of unobtrusive notifications 
increase the motivation of participants of a meeting to actively ventilate a room.

\section{BACKGROUND}

Air Quality in Meeting Rooms. Air quality in a closed meeting room improves with decreasing concentrations of air components that negatively affect the cognitive performance of participants. Smart technology can be used to determine the concentration of various gases, as demonstrated by Dang et al. [7]. For the assessment of air quality in this paper, we limit the scope to carbon dioxide $\left(\mathrm{CO}_{2}\right)$, since the concentration of $\mathrm{CO}_{2}$ in a meeting room increases continuously with the duration of the meeting due to the breathing of participants. Other gases that can be measured (e.g., methane, propane, ammonia, carbon monoxide, nitrogen dioxide), usually are not relevant during a meeting.

Official government documents of Germany [1] state three $\mathrm{CO}_{2}$ concentrations (inconspicuous: $<1000 \mathrm{ppm}$, conspicuous: 1000 2000 ppm, unacceptable: $>2000$ ppm) that are based on studies conducted before 2008. In terms of research literature, Azuma et al. [4] documented works that explored and discussed the effects of $\mathrm{CO}_{2}$ on human body from which we discuss the most related studies. In most of the studies, the cognitive performance of participants were reduced with higher concentration of $\mathrm{CO}_{2}$, where the studies investigated different concentrations (i.e., Satish et al. [18]: 1000 ppm, Allen et al. [3]: $950 \mathrm{ppm}$, Maula et al. [14]: $2260 \mathrm{ppm}$ ). For pilots, Allen et al. [2] found in a study that at a concentration of $2500 \mathrm{ppm}$ the performance of the pilots was significantly lower compared to $700 \mathrm{ppm}$. However, there is also a study [22] that could not find significant deterioration of cognitive performance up to $3000 \mathrm{ppm}$. Ambient Light Notifications. Ambient notifications have their origins in an early work by Mark Weiser [21] in which he began a dialogue about "Calm technology". In his work, he discussed "Dangling String", which consisted of a 8 foot piece of plastic spaghetti hanging from the ceiling and informing about the network traffic via an electric motor. The piece of plastic informs without being obtrusive by moving from peripheral attention to the center of attention of the user. Later, Hiroshi Ishii from the MIT Media Laboratory presented the ambientRoom [10], which, similar to the work of Mark Weiser, integrated different types of ambient notification displays, including ambient lighting, to study peripheral attention. Since then, many different ambient notification types have been explored. In the following, we concentrate the discussion on studies and systems related to ambient lighting for notifications which motivate people to become physically active.

Mueller et al. [15] explored eight different ambient light designs by means of LED stripes that were mounted behind a computer monitor. These included designs with sine-waves of different frequencies and gradual changes of parameters affecting saturation, brightness and color. Their study investigated how well the different designs can reflect a period of time by an expiring ambient light timer, hence the name "AmbientTimer". Results show that changes of complementary colors are superior to changes of adjacent colors and quick sine-wave changes of the parameters were more distracting than gradual changes. We use their results in the design of our notifications to determine if more noticeable notification types are more likely to result in activity.
The system "MoveLamp" from Fortmann et al. [8] investigated whether ambient light notification can motivate users to be more physically active. It consisted of an ambient lighting system, which indicated the amount of activity of a user by the color and brightness from green to red. When it was green, the activity battery was fully charged, so to speak, and when it was red, the activity battery was empty. The amount of activity was measured by means of pedometers. By using the system, their study has shown that the system helps people to move more and more often during a working day. The colors used in the system and the goal of increasing activity are similar to those in our study, although our notifications do not indicate a property of the user, but a property of the environment.

Similar to "MoveLamp“, "Pediluma“ by Lim et al. [12] seeks to increase physical activities of the user by means of ambient lighting. Instead of an ambient lamp, the ambient light was indicated by the brightness of green light on a shoe accessory. Results also indicate that the system was also able to increase the activity of a user. Maan et al. [13] utilizes ambient light to influence the behavior of users in terms of energy consumption (i.e., central heating). A high power LED lamp was used to project ambient light onto a wall, which blended from green, yellow, to red, depending on energy usage. They found that ambient light feedback is easier to process than numerical feedback and that ambient light has a positive effect on energy efficiency. Our work pursues a similar goal but in a different domain (air quality), whereby users in our system would have to perform more physical actions (instead of adjusting a heating controller). In the realm of smart technology, Seiderer et al. [20] used ambient light notifications to indicate open windows and doors at opportunistic locations within a house. They also used the colors green and red and were able to show that house inhabitants quickly understood this type of notification and its meaning. The provided notifications helped the residents to improve the so-called "peace of mind".

Roumen et al. [17] studied different notification channels including ambient lighting by means of a wearable interactive ring called NotiRing during physical activity. Their results indicate that on a ring, ambient lighting was less noticeable while vibrations was the most reliable and fastest channel to convey notifications. Hence, we included the vibration channel in our work, however, in combination with ambient lighting notifications in the surrounding environment. In a perceptibility study, Komninos et al. [11] investigated smartphone notifications together with ambient lighting. They conducted two experiments that simulated multi-tasking of users in a smarthome environment which is similar to our smart meeting room context. In the first study, the different notification channels of a smartphone (audio, vibration, device LED) in every possible combination and also together with ambient lighting were implemented and the response times in which users perceived the notifications were measured. In the second study, four different types of ambient light notifications (permanent on, blink twice, slow pulsing, rapid pulsing) were compared, with blinking twice and rapid pulsing showing the shortest reaction times. The authors found that the audio channel has the best response times for perceptibility and response times of every other combination could be reduced by adding the audio channel. The same was measured for ambient lighting even though the audio channel reduced the response times a bit more. In our meeting room context, however, 
the audio channel as well as rapid pulsing ambient light would be too obtrusive for air quality notifications and would potentially disturb meetings too much. It is therefore very promising that the same effect can be achieved with ambient lighting [11].

\section{SMART MEETING ROOM DESIGN}

The system for our study was installed in a meeting room without automatic ventilation. Figure 1 gives an overview of the room with the placement of the components. The hardware setup (i.e., the components) was similar to the one presented in [19]. In the following, we explain the most important functions required for the study. Next to all openable windows (W1 to W5 in Figure 1), RGB-LED stripes were placed which are controlled via WiFi-enabled ESP8266microcontrollers by a Raspberry Pi 3 B + single-board computer. The system provides an automatic mode in which wireless contact sensors on each window and door, a motion detector, and $\mathrm{CO}_{2}$ air quality sensors are utilized. In this mode, our open-source software "Eventerpretor" 2 controls the LED stripes based on a rule system. All LED stripes are switched off if no motion has been detected by the motion sensor for 15 minutes. If motion is detected, all LED stripes are switched on and their color and brightness pattern is adapted. The color of the LED stripes depends on the five minutes averaged $\mathrm{CO}_{2}$ concentration measured in this room. Red color indicates a concentration level higher than $2000 \mathrm{ppm}$, yellow color a level between $1000 \mathrm{ppm}$ and $2000 \mathrm{ppm}$, and green color a level below $1000 \mathrm{ppm}$. During the study the automatic adaptation of the ambient lights (color and brightness) was switched off. Instead, the behavior of the LED stripes was controlled manually by an experimenter using a web interface.

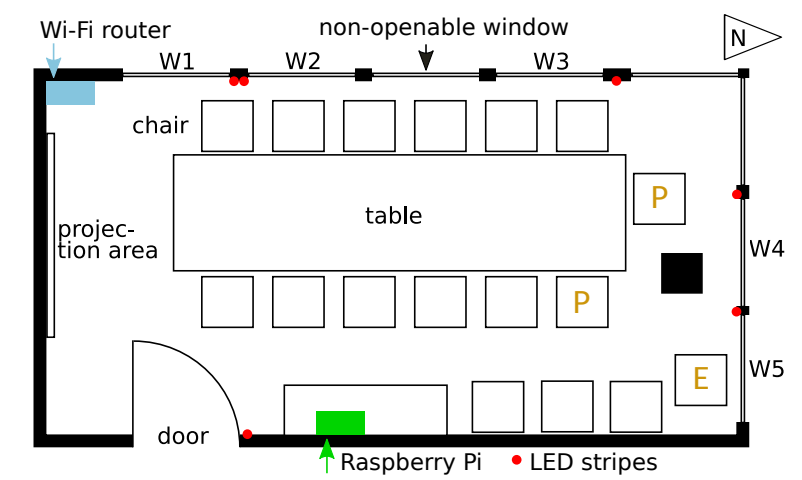

Figure 1: Overview of the meeting room. The chairs' positions of the study participants are labeled with a "P", the experimenter's chair with " $E$ "

\section{STUDY}

The purpose of the study was to investigate the perceptual capacity of different notification types, which were implemented by ambient lighting and the vibration of a smartwatch. The study also investigated whether the type of notification has an influence on the motivation to react directly to an event, that is to open a window in

\footnotetext{
${ }^{2}$ https://github.com/andreas-seiderer/Eventerpretor
}

order to improve the air quality. Furthermore, we were interested in whether personality has an influence on whether one tends to actively improve air quality in such a situation. For this purpose, we measured personality traits by means of the Big-Five inventory questionnaire by Rammstedt [16], which measures openness, conscientiousness, extraversion, agreeableness, and neuroticism.

The research question we aimed to study was how notification type influences users' performance of perceiving a message and their willingness to open a window during a work meeting. We were also interested in any influences of personality, assuming, for example that less extravert users would be less willing to open a window. We chose three notification types which are denoted with the terms Permanent, Pulsing, and Smartwatch. In general, the LED stripes were permanently lit in green to indicate normal air quality. If the first condition Permanent was activated, the color of the LED stripes immediately changed to red and remained red until the normal state was activated. The second condition Pulsing caused the LED stripes to pulse in red. That is, the brightness of the LED stripes faded repeatedly with a period of 3.5 seconds from bright to darker and then back to bright again, whereby the LED stripes in the darkest state still shined with low intensity. According to $[11,15]$, too rapid changes in parameters, such as brightness, have been shown to be more distracting than gradual changes, which would interrupt or disturb processes or the concentration of participants in meetings. Therefore, we chose a noticeably different type of notification for the second condition, whereby the slow pulsing greatly alleviated the distracting character. Slow pulsing is reminiscent of breathing instead of the alarm character of rapid pulsing. The third condition Smartwatch made use of the unobtrusive vibration of a smartwatch and was based on the second condition Pulsing. In addition to the pulsating red LED stripes, the smartwatch received a message and vibrated once discreetly for half a second.

\subsection{Design and Tasks}

The study design placed participants in the context of a meeting in which they listened to a speaker in a concentrated manner with at least one additional meeting participant. The latter was necessary for a realistic picture of the perception of a participant, since meetings usually take place with at least one other person and this person may influence the perception of ambient light notifications. In order to create a realistic situation that can be repeated in each session, we adopted the study design approach of Maan et al. [13] and let participants perform an adequate cognitive load task that replicates a task that also occurs in meetings.

Primary Task. Hence, as the primary task, participants had to listen to a speaker and answer questions about the talk on a notebook in front of them, c.f., Figure 3. To achieve reproducibility of the talk and its speaker, a TED talk (17:54 minutes, TEDxRheinMain 2012, "Das Ende des eigenen Autos", Michael Huebl) was chosen and shown on the projection screen in the meeting room. During the talk, participants had to complete a quiz as good as possible. Questions about the talk were displayed on the notebook at predetermined times, c.f, Figure $2 b$, which matched the course and content of the talk. A question was presented to participants until either the question was answered or until it was time for the next 


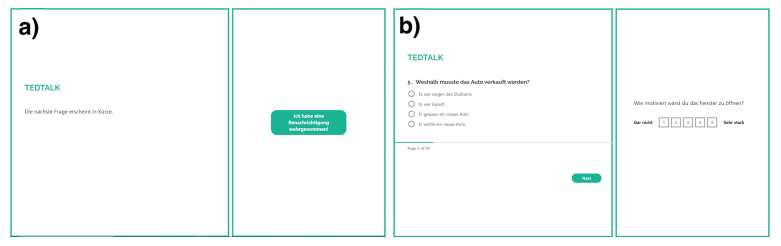

Figure 2: Two screenshots of the study UI showing the questions of the quiz and notification confirmation area.

question. When a question was answered, the question disappeared and a wait prompt was displayed, c.f, Figure 2a. Participants had to follow the talk in order to choose the right answer from the given options, c.f, Figure 2b. In total there were 29 questions spread over 18 minutes. Participants were offered sweets and coffee as a reward for successfully completing the quiz.

Secondary Task. During the talk, all 3 notification types were presented one after the other at intervals of 5 minutes to signal poor air quality. After 60 seconds, the notifications were deactivated and the LED stripes were lit with green color. The sequences of the notification types were counter-balanced and distributed over 9 sessions to which the participants were randomly assigned. As the secondary task, participants had to click on a button on the study UI, c.f, Figure 2a, as soon as they have noticed the notification. At that same time, participants had to indicate on a scale (1 - not at all ... 5 - very highly) how motivated they were to get up and open a window at that moment, c.f, Figure $2 b$. The study UI then logged the response time for the presented notification type and the given motivation rating. This utilizes the Experience Sampling Method $[6,9]$ to query the participants' experience at the moment of occurrence.

\subsection{Participants and Study Set-up}

For our study, we recruited 18 participants (7 female) which were mostly university students and staff from the fields of business informatics, computer science, primary school teaching, or journalism. The participants age varied between 20 and 34 years with an average of 25.24 years $(\mathrm{SD}=4.12)$. All participants were right-handed and 11 of them already had experience with a smartwatch. Figure 1

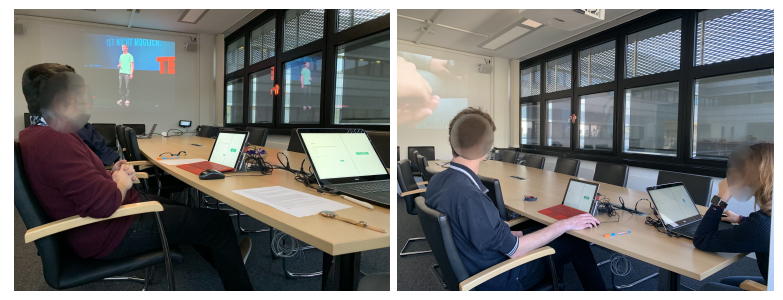

Figure 3: Participants following the TED talk and their placement in the meeting room.

sketches the positions of the participants $(\mathrm{P})$ and the experimenter (E), who was placed inconspicuously in the background behind the participants. In the room, the (slightly transparent) blinds were lowered so that participants were not distracted (as much as possible) by external events.

In front of each participant, a notebook was placed on the table on which the study UI was running. Nothing else was placed on the table except the PC to play the TED talk. The study UI was implemented as a nodeJS server application running on the notebooks, which communicated via WiFi with the experimenter's PC, generated the study UI as HTML/JavaScript, and logged all events (e.g. mouse clicks, answers to questions, acknowledgment of notifications, timestamps, etc.) locally. Both notebooks (Microsoft Surface Pro 3, Dell Precision M3800) had a mouse connected with which the study UI, c.f, Figure 2, had to be operated. Both participants had a clear view on their notebook as well as the projection screen from their positions. At the same time participants could see several LED stripes, which were positioned at the windows, c.f, Figure 3.

\subsection{Procedure}

Each session always required two participants, thus we invited the participants accordingly. On arrival in the meeting room, participants were welcomed by an experimenter and requested to sign a data protection form (GDPR) and fill out a questionnaire. The questionnaire included demographic data (e.g. gender, age, handedness, experience with smartwatches) and contained 10 questions on personality from the Big-Five inventory [16]. Afterwards, participants were given a document to read through, which contained an introduction to the study, a description of the smart equipment and ambient lighting, and the procedure of the study. Thus, participants knew which task should be carried out, what they had to do, and what the colors of the ambient lighting meant. They also knew that the smartwatch only vibrates and nothing else had to be done with the smartwatch. After questions of participants were answered and participants confirmed that everything was understood, each participant was given a smartwatch, which they put on their dominant hand or instead of a watch already worn. After that, the TED talk was started and the study UI on the notebooks in front of the participants started with the questions of the quiz. During that time, the three notification types were presented according to the previously determined sequence at 5 minute intervals. An experimenter observed the experiment in the background and took written notes on the behavior of the participants. After each session, participants were invited to sweets and coffee as a reward and to talk about their experiences. Each of the sessions including discussion lasted between 40 and 60 minutes.

\subsection{Results}

During all sessions, we could observe that the selected primary task (TED talk) achieved the goal of focusing attention as well as inducing cognitive load. Of 522 quiz questions presented, 95\% (496) were answered and of the questions answered, 92.5\% were answered correctly. On average, it took 8.24 seconds $(\mathrm{SD}=0.07)$ from the presentation of a question to the submission of an answer. When the speaker made funny statements, emotional reactions such as laughter were expressed by participants in all sessions. This indicates that the cognitive load of the participants was high and the participants followed the talk attentively. Overall, the primary task 
was able to attract the attention of participants, causing the ambient displays to be moved to the peripheral perception of participants. All participants chose the left arm to wear the smartwatch. SPSS skipped one participant's data from the statistical analysis because the participant had completely missed a notification and therefore no response time was measured and no rating was given. Figure 4

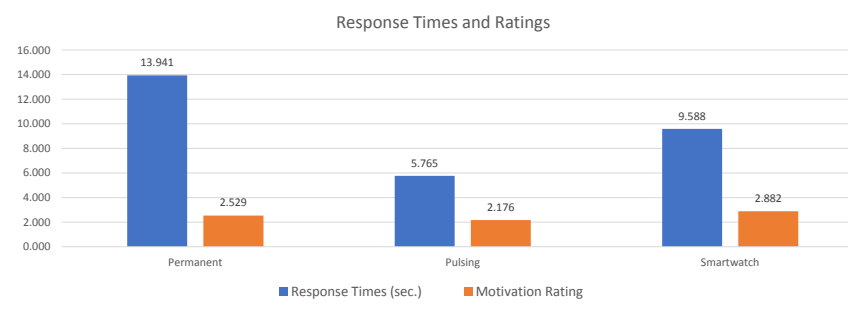

Figure 4: Response times (sec.) and ratings on motivation.

shows for all three notification types the measured response times (seconds to perceive a notification) and ratings for the motivation to open a window. Pulsing was perceived fastest with an average of 5.77 seconds $(S D=5.506)$, followed by Smartwatch with 9.59 seconds $(S D=7.649)$. The type Permanent was recognized worst with an average of 13.94 seconds $(S D=13.193)$.

At first glance, the average response time for the condition Smartwatch seems to be too high, since it was based on condition Pulsing in the ambient lighting mode and therefore should hardly deviate from it. The notes of the experimenters show that at least 6 participants first interacted with the smartwatch's notification center to read the notification on the smartwatch display before they confirmed the perceived notification on the study UI. The observed behavior was unexpected because participants were instructed in advance that immediate confirmation was important and were informed that no interaction with the smartwatch was necessary. We believe that the primary task (TED talk, quiz) attracted the attention so much that participants showed a natural reaction to the vibration of the smartwatch and therefore they first checked the display on the smartwatch. This confirms the obtrusive character of the vibration channel $[11,17]$ and may be too obtrusive for the meeting room context with ambient lighting as it seems to have a rather dominant effect on participants' attention and may prevent the ambient lighting from quickly moving to the center of attention.

A repeated measurement ANOVA on the measured response times showed significant differences between the notification types $F(2,32)=3.49, p=.043$, partial $\eta=.179$. The post-hoc pairwise comparisons (with Bonferroni corrections) for each measured notification type revealed that there are significant differences between Permanent and Pulsing ( $p=.034,8.176,95 \%-C I[0.539,15.814])$ with the ambient notification through Pulsing as significantly faster perceivable as Permanent.

Figure 4 also shows the ratings for motivation to open a window for all three notification types. The condition Smartwatch was rated highest with an average of $2.88(S D=1.495)$, followed by Permanent with $2.53(S D=1.505)$. The type Pulsing was rated worst with an average of $2.18(S D=1.286)$. The median value of the scale is 3 and even though some participants were highly motivated (rated 5) to open a window for certain notifications and less for others, the averages show that our participants were rather restrained $(<3)$ in their general motivation. Here, a repeated measurement ANOVA showed significant differences between the ratings $F(2,32)=3.963, p=.029$, partial $\eta=.199$. However, the post-hoc pairwise comparisons (with Bonferroni corrections) for ratings for each measured notification type did not reveal clearly significant different conditions. Only Pulsing and Smartwatch show a low significance level of $p=.054, .706,95 \%-C I[-.010,1.422]$.

The personality of participants was measured in the initial questionnaire on the basis of the dimensions openness, conscientiousness, extraversion, agreeableness, and neuroticism [16]. In particular, conscientiousness and extraversion was relevant to our research questions. However, Pearson correlation tests across all personality dimensions in combination with motivation ratings on all three notification types could not confirm a strong correlation.

\section{DISCUSSION}

We are living in an automated information society in which many daily interactions are becoming automated. While it is easy to sense information, such as sensing air quality in a room, automating physical interactions (e.g., opening and closing the window) is still cumbersome and often associated with high costs. Ideally, meeting rooms should be equipped with perfectly automated air conditioning systems to improve wellbeing and health. The reality is that most buildings are not equipped with such technology and human action is needed.

In this paper, we have studied the effect of different unobtrusive (low-cost) modalities to display air quality on participants' performance in perceiving the need to open a window, and their motivation to act on this perceived information. In our study, we have observed a significant effect of interaction modality on efficiency of informing participants. They were fastest to recognize bad air quality in the Pulsing condition and we also found that the usage of the Smartwatch condition seems to interfere with the ambient light displays, ultimately providing no added value but potential for confusion. Based on our observations and discussions with participants, we believe it is important that air quality displays in a social setting are visible for all potential users in the room to also inform others why a person in the room would (want to) open the window.

Considering an effect of modality and personality on motivation, our study did not find a significant result. We believe that related variability may be too large and a much larger study is needed to explore related effects. However, our results and following insights may still help to optimize future research on this aspect as well. In our post-study discussion with the small group of participants, they told us that there were "no punishment" for not wanting to open a window and that they themselves did not experience the bad air quality. Therefore, participant did not experience intrinsic motivation (e.g., live healthy) or extrinsic motivation (e.g., get a reward or prevent punishment). In contrary, they have argued that in the study setup they felt as if they were to open the window they would be missing out on the presentation and the questions they were asked to answer, which they perceived as of higher priority. We believe that to this end future studies could adjust the (perceived) priority of tasks and integrate a reward-punishment system. 
Our research is partly motivated by Calvo and Peters' work on positive computing [5]. They argue that technology design needs to address the psychological wellbeing of users and aim to support their individual development through, for example, improving their competence. We believe that air quality displays could improve long-term participants' awareness and influence their motivation to open windows to improve air quality. We have had an ambient air display integrated in a meeting room for more than a year and based on our own "autobiographic" experience, we can tell that long-term exposure to ambient air quality displays does sensitize and change one's awareness and behavior, becoming willing to open windows.

In order to speed up such expected change in behavior, we want to propose, based on our study's results, to first implement a solution that will be efficient in conveying air quality information (i.e., implementing a technique similar to Pulsing condition). After some time the system could change its behavior towards implementing a more ambient technique such as the Permanent condition. Implementing a condition such as Smartwatch would only be beneficial if most of the room users are guests and there are a few people who usually open windows.

\section{CONCLUSION}

Air quality information can be sensed and displayed easily indoors with today's IoT technologies. But displaying such information to participants of a meeting in a manner that they will perceive the information without disturbing them during important work is a challenge. Moreover, displaying the information in a technique that potentially motivates to open a window for health reasons would be desirable. In this paper, we presented a user study exploring the effect of three air quality information techniques on participants' performance in perceiving the information and their motivation to act on this information. We reported significant difference of display modality on performance and argued that to effect participants' motivation more context information needs to be considered. Moreover, we suggest adapting the system's display technique over time to speed up a health beneficial change in participants' behavior. We hope that our research provides knowledge and inspirations to progress the research field of ambient displays for health and to ultimately improve the design of new air quality displays.

\section{ACKNOWLEDGMENTS}

We thank the students Carina Preissler, David Kurz, and Marc Blickle for their contribution to this work.

\section{REFERENCES}

[1] -. 2008. Gesundheitliche Bewertung von Kohlendioxid in der Innenraumluft. Bundesgesundheitsblatt - Gesundheitsforschung - Gesundheitsschutz 51, 11 (01 Nov 2008), 1358-1369.

[2] Joseph G Allen, Piers MacNaughton, Jose Guillermo Cedeno-Laurent, Xiaodong Cao, Skye Flanigan, Jose Vallarino, Francisco Rueda, Deborah Donnelly-McLay, and John D Spengler. 2018. Airplane pilot flight performance on 21 maneuvers in a flight simulator under varying carbon dioxide concentrations. Fournal of exposure science \& environmental epidemiology (2018), 1.

[3] Joseph G Allen, Piers MacNaughton, Usha Satish, Suresh Santanam, Jose Vallarino, and John D Spengler. 2016. Associations of Cognitive Function Scores with Carbon Dioxide, Ventilation, and Volatile Organic Compound Exposures in Office Workers: A Controlled Exposure Study of Green and Conventional Office Environments. Environmental Health Perspectives 124, 6 (2016), 805.
[4] Kenichi Azuma, Naoki Kagi, U. Yanagi, and Haruki Osawa. 2018. Effects of low-level inhalation exposure to carbon dioxide in indoor environments: A short review on human health and psychomotor performance. Environment International 121 (2018), 51 - 56.

[5] Rafael A Calvo and Dorian Peters. 2014. Positive computing: technology for wellbeing and human potential. MIT Press.

[6] Sunny Consolvo and Miriam Walker. 2003. Using the Experience Sampling Method to Evaluate Ubicomp Applications. IEEE Pervasive Computing 2, 2 (April 2003), 24-31. https://doi.org/10.1109/MPRV.2003.1203750

[7] Chi Tai Dang, Andreas Seiderer, and Elisabeth Andre. 2018. Theodor: a step towards smart home applications with electronic noses. In Proceedings of the 5th international Workshop on Sensor-based Activity Recognition and Interaction iWOAR '18: Berlin, Germany - September 20 - 21, 2018. https://doi.org/10.1145/ 3266157.3266215

[8] Jutta Fortmann, Tim Claudius Stratmann, Susanne Boll, Benjamin Poppinga, and Wilko Heuten. 2013. Make Me Move at Work! An Ambient Light Display to Increase Physical Activity. In Proceedings of the 7th International Conference on Pervasive Computing Technologies for Healthcare (Venice, Italy) (PervasiveHealth '13). ICST (Institute for Computer Sciences, Social-Informatics and Telecommunications Engineering), Brussels, BEL, 274-277. https://doi.org/10.4108/icst. pervasivehealth.2013.252089

[9] Joel Hektner, Jennifer Schmidt, and Mihaly Csikszentmihalyi. 2006. Experience Sampling Method: Measuring the Quality of Everyday Life.

[10] Hiroshi Ishii, Craig Wisneski, Scott Brave, Andrew Dahley, Matt Gorbet, Brygg Ullmer, and Paul Yarin. 1998. AmbientROOM: Integrating Ambient Media with Architectural Space. In CHI 98 Conference Summary on Human Factors in Computing Systems (Los Angeles, California, USA) (CHI'98). Association for Computing Machinery, New York, NY, USA, 173-174. https://doi.org/10.1145/286498.286652

[11] Andreas Komninos, Jeries Besharat, Vassilios Stefanis, Georgia Gogoulou, and John D. Garofalakis. 2019. Assessing the perceptibility of smartphone notifications in smart lighting spaces. FAISE 11, 3 (2019). https://doi.org/10.3233/AIS-190525

[12] Brian Y. Lim, Aubrey Shick, Chris Harrison, and Scott E. Hudson. 2010. Pediluma: Motivating Physical Activity through Contextual Information and Social Influence. In Proceedings of the Fifth International Conference on Tangible, Embedded, and Embodied Interaction (Funchal, Portugal) (TEI'11). Association for Computing Machinery, New York, NY, USA, 8. https://doi.org/10.1145/1935701.1935736

[13] Saskia Maan, Bo Merkus, Jaap Ham, and Cees Midden. 2011. Making it not too obvious: the effect of ambient light feedback on space heating energy consumption. Energy Efficiency 4, 2 (01 May 2011). https://doi.org/10.1007/s12053-010-9102-6

[14] H Maula, V Hongisto, V Naatula, A Haapakangas, and H Koskela. 2017. The effect of low ventilation rate with elevated bioeffluent concentration on work performance, perceived indoor air quality, and health symptoms. 27, 6 (2017).

[15] Heiko Müller, Martin Pielot, Wilko Heuten, Anastasia Kazakova, and Susanne Boll. 2012. Unobtrusively Reminding Users of Upcoming Tasks with Ambient Light: Ambient Timer. In Proceedings of the 7th Nordic Conference on HumanComputer Interaction: Making Sense Through Design (Copenhagen, Denmark) (NordiCHI '12). Association for Computing Machinery, New York, NY, USA, 801-802. https://doi.org/10.1145/2399016.2399155

[16] Beatrice Rammstedt and Oliver P. John. 2007. Measuring personality in one minute or less: A 10-item short version of the Big Five Inventory in English and German. Fournal of Research in Personality 41, 1 (2007), 203 - 212. https: //doi.org/10.1016/j.jrp.2006.02.001

[17] Thijs Roumen, Simon T. Perrault, and Shengdong Zhao. 2015. NotiRing: A Comparative Study of Notification Channels for Wearable Interactive Rings. In Proceedings of the 33rd Annual ACM Conference on Human Factors in Computing Systems (Seoul, Republic of Korea) (CHI '15). Association for Computing Machinery, New York, NY, USA, 2497-2500. https://doi.org/10.1145/2702123.2702350

[18] Usha Satish, Mark J Mendell, Krishnamurthy Shekhar, Toshifumi Hotchi, Douglas Sullivan, Siegfried Streufert, and William J Fisk. 2012. Is CO2 an indoor pollutant? Direct effects of low-to-moderate $\mathrm{CO} 2$ concentrations on human decision-making performance. Environmental health perspectives 120, 12 (2012), 1671.

[19] Andreas Seiderer, Ilhan Aslan, Chi Tai Dang, and Elisabeth Andre. 2019. Indoor air quality and wellbeing - enabling awareness and sensitivity with ambient IoT displays. Lecture Notes in Computer Science 11912 (2019), 266 - 282. https: //doi.org/10.1007/978-3-030-34255-5_18

[20] Andreas Seiderer, Chi Tai Dang, and Elisabeth Andre. 2017. Exploring opportunistic ambient notifications in the smart home to enhance quality of live. In Enhanced quality of life and smart living: 15th International Conference, ICOST 2017, Paris, France, August 29-31, 2017, Mounir Mokhtari, Bessam Abdulrazak, and Hamdi Aloulou (Eds.). https://doi.org/10.1007/978-3-319-66188-9_13

[21] Mark Weiser. 2004. Designing Calm Technology. https://calmtech.com/papers/ designing-calm-technology.html

[22] Xiaojing Zhang, Pawel Wargocki, Zhiwei Lian, and Camilla Thyregod. 2017. Effects of exposure to carbon dioxide and bioeffluents on perceived air quality, self-assessed acute health symptoms, and cognitive performance. 27, 1 (2017). 